\title{
Estudio sobre Automedicación en Población Universitaria Española
}

\author{
Patricia Guillem Sáiza, Francesc Francès Bozala, \\ Francisco Gimenez Fernández ${ }^{\mathrm{a}}$, Carmen Sáiz Sánchez ${ }^{\mathrm{a}}$
}

\begin{abstract}
a Departamento Medicina Preventiva i Salut Pública, Ciències de l'Alimentació, Toxicologia i Medicina Legal. Universitat de València.
\end{abstract}

\section{Correspondencia:}

Francesc Francès Bozal, Departamento Medicina Preventiva i Salut Pública, Ciències de l'Alimentació, Toxicologia i Medicina Legal, Universitat de València, Avda. Blasco Ibáñez n 15, 46010 - València, España. Telf.: 96 3983107, correo electrónico: ffrances@uv.es

Recibido el 22 de marzo de 2010.

Aceptado para su publicación el 14 de junio de 2010.

\begin{abstract}
RESUMEN
Objetivo. La automedicación es una práctica común en diferentes sociedades que puede tener importantes implicaciones sanitarias. Esta práctica ha sido poco estudiada hasta ahora en población universitaria española. Nuestro objetivo es cuantificar esta actividad y estudiar los factores asociados a la misma.

Material y método. Se diseñó y facilitó un cuestionario para su autocumplimentación a una muestra de población universitaria. Se evaluó el impacto de tres variables principales sobre la automedicación: sexo, estudios sanitarios y hábitos de información sobre medicamentos. Se emplearon en el análisis los test de Chi Cuadrado, junto a técnicas de regresión logística.

Resultados. La práctica de automedicación fue confirmada en el 90,8\% de los 501 individuos que contestaron correctamente la encuesta. Esta práctica fue significativamente más frecuente en mujeres. El grupo de medicamentos más frecuentemente consumido fueron los analgésicos/antiinflamatorios (consumidos por el $73 \%$ de los automedicadores). Las mujeres consumieron significativamente más anticatarrales $(O R=1,70)$, mientras que los hombres consumieron más antibióticos $(\mathrm{OR}=1,88)$. Los individuos con hábitos de información sobre medicamentos presentaron un menor consumo de antibióticos $(\mathrm{OR}=2,15)$.

Conclusiones. La práctica de la automedicación es elevada en población universitaria valenciana y mayor de la reportada anteriormente en nuestro país. El sexo y los hábitos de información están asociados a la automedicación en determinados grupos de medicamentos.

Palabras clave. Automedicación, Estudiantes, Universidad.
\end{abstract}

\section{ABSTRACT}

Study on self-medication in the Spanish university population

Aims. Self-medication is a common behaviour that can have serious effects on health. This practice has been little studied in Spanish university population. Our aim is to assess the importance of this behaviour and to study its associated factors.

Material and methods. A self-completed questionnaire was designed and administered to a sample of Spanish university students. The impact of sex, type of studies and drug information habits was assessed. Chi-square and logistic regression models were undertaken. Results. Self-medication was confirmed in $90.8 \%$ of the 501 individuals that correctly answered the questionnaire. This practice was significantly more common among women. The analgesic/anti-inflammatory group of drugs was the most frequently consumed $(73 \%$ of self-medicators). Women significantly consumed more anticatarrhals $(\mathrm{OR}=1.70)$, whereas antibiotics were more consumed by men $(\mathrm{OR}=1.88)$. Furthermore, individuals with good drug information habits had less antibiotic consumption $(\mathrm{OR}=2.15)$.

Conclusions. Self-medication is a very common habit in Valencian university population, and is the highest reported to date in our country. Sex and drug information habits are associated with self-medication in some groups of drugs.

Key words. Self Medication, Students, University.

\section{INTRODUCCIÓN}

La automedicación puede ser definida de forma genérica como la administración por decisión propia, o por consejo no cualificado, de medicamentos para aliviar un síntoma o curar una enfermedad ${ }^{1}$. Este comportamiento está ampliamente extendido a nivel mundial, con cifras alrededor del $60 \%$ en países tan dispares económica y culturalmente como España o la India ${ }^{2,3}$. Esta elevada frecuencia puede ser atribuida tanto a la presencia cotidiana de los medicamentos en dichas sociedades, como en ocasiones a la escasa cobertura en los servicios sanitarios ${ }^{4,5}$. 
En nuestro país algunos medicamentos se pueden adquirir sin prescripción médica por estar clasificados por la Dirección General de Farmacia y Productos Sanitarios como "especialidades publicitarias". Este tipo de medicamentos, de venta libre, en el caso de EEUU supone el $40 \%$ de las medicinas consumidas $^{6}$.

Múltiples factores han sido implicados en el autoconsumo de medicamentos. Entre ellos cabe citar la edad, el sexo, la orientación hacia el autocuidado y el conocimiento de la medicación $n^{7,8,9}$.

Dado que, aunque existen estudios relativos a las conductas de automedicación en población universitaria en otros países ${ }^{10-13}$, existen pocos datos relativos a este comportamiento en población universitaria española, el propósito de nuestro estudio es estimar el nivel de automedicación relacionado con trastornos ligeros de salud en un colectivo universitario, así como los factores asociados a este comportamiento.

\section{MATERIAL Y MÉTODO}

En este estudio transversal se obtuvieron datos de un total de 501 estudiantes universitarios de València, con edades comprendidas entre 18-28 años que cursaban estudios de Medicina, Odontología, Relaciones Laborales, Ciencias de la Alimentación, Nutrición y Veterinaria.

Se diseñó un cuestionario de cuatro secciones. La mayoría de preguntas eran de carácter cerrado, de manera que el participante únicamente tenía que seleccionar una opción. En la primera sección se recogían datos de carácter sociodemográfico como edad, sexo, tipo de estudios en curso, procedencia, etc. La segunda sección versa sobre los problemas de salud e incluye tanto una cuestión cerrada sobre la autopercepción del nivel de salud, como ítems sobre la utilización de recursos sanitarios, principales problemas de salud que se padecen, reacciones adversas a medicamentos, estilos de vida, etc. El tercer subgrupo y pieza clave del cuestionario es el grupo de ítems relativos al consumo de medicamentos sin prescripción e indicación médica. En este grupo se diferenciaron las especialidades farmacéuticas publicitarias de las especialidades con receta médica, y se sistematizaron en categorías.

La última sección del cuestionario abordaba la información sobre el medicamento. Se valoró la permeabilidad de los individuos ante los medios publicitarios e informativos (recuerdo de especialidades publicitadas en medios de comunicación, conocimiento de las campañas del Ministerio de Sanidad sobre prescripción de medicamentos y uso racional de antibióticos) y el grado de conocimientos relativos al uso responsable del medicamento (lectura del prospecto, comprensión, razones de uso, informe de RAM). Este último parámetro se valoró me- diante un score final entre 0 y 10 puntos, resultado de la suma de las puntuaciones obtenidas en cada fuente de información sobre el medicamento por la que era interrogado ( 0 no se informa, 1 a veces, 2 a menudo/siempre). Se dicotomizó posteriormente esta variable de score en nula-escasa información (0-5) y buena información (6-10). Todos los participantes otorgaron su consentimiento informado.

Los datos correspondientes a las variables recogidas en cada ítem se codificaron e introdujeron en una base de datos y fueron analizados mediante el programa estadístico SPSS versión 15. El estudio descriptivo se expresó mediante medias, porcentajes y media $\pm D E$. En el posterior estudio de asociación se utilizó la t Student para grupos independientes para la comparación de medias en variables continuas. Para la comparación de porcentajes se utilizó la Chi-cuadrado de Pearson. Por último, se emplearon técnicas de regresión logística binaria y múltiple para la obtención de modelos multivariantes.

\section{RESULTADOS}

Un total de 501 encuestas fueron correctamente cumplimentadas y retornadas. La edad media de nuestra muestra fue $20,4 \pm 2,3$ años. La tabla 1 muestra los porcentajes de automedicación en función de diferentes variables registradas en el cuestionario. Es de destacar que más de las 3 cuartas partes de los estudiantes son mujeres. También es de destacar que únicamente un $9,6 \%$ de los encuestados refirieron tener un estado de salud malo o muy malo.

El $90,8 \%$ de los entrevistados realizaba prácticas definidas como automedicación. Este porcentaje fue significativamente mayor en las mujeres $(92,7 \%$ frente a $84,7 \%, p=0,009$ ). No se evidenciaron diferencias significativas entre estudiantes de carreras sanitarias y no sanitarias en lo referente a la automedicación (88,9\% frente a $91,6 \%$, respectivamente, $p=0,342$ ). No obstante, existieron diferencias de porcentaje de automedicación cercanas a la significación estadística en función de los estudios sanitarios o no de los progenitores, siendo los hijos de padres no sanitarios los que presentaron niveles mayores de automedicación $(86,3 \%$ de automedicadores en hijos de padres sanitarios frente a $91,98 \%$ en progenitores no sanitarios, $p=0,09$ ).

Los analgésicos fueron el grupo de fármacos más usado, siendo consumido por el $73,8 \%$ de los automedicadores. El segundo grupo de fármacos más utilizado fueron los anticatarrales $(44,1 \%)$, seguidos de los antidepresivos (13,4\%) y los antibióticos $(12,2 \%)$.

Muchos individuos tomaban más de un medicamento simultáneamente. Así, el 25,5\% tomaba únicamente un solo medicamento, el 33,9\% consumía dos medicamentos a la vez, el 21,5\% tomaba tres 


\begin{tabular}{llcc}
\hline VARIABLE & & NÚMERO (\%) & AUTOMEDICACIÓN (\%) \\
\hline Sexo & Hombre & $118(23,6 \%)$ & $100(84,7 \%)$ \\
& Mujer & $383(76,4 \%)$ & $355(92,7 \%)$ \\
Estudios sanitarios & $\mathrm{Sí}$ & $357(71,3 \%)$ & $327(91,6 \%)$ \\
& $\mathrm{No}$ & $144(28,7 \%)$ & $128(88,9 \%)$ \\
Profesión sanitaria & $\mathrm{Si}$ & $95(18,9 \%)$ & $82(86,3 \%)$ \\
de progenitor & $\mathrm{No}$ & $406(81,1 \%)$ & $373(91,9 \%)$ \\
Estado de salud & Bueno/Muy bueno & $453(90,4 \%)$ & $410(90,5 \%)$ \\
& Regular/Malo/Muy malo & $48(9,6 \%)$ & $45(93,8 \%)$ \\
Se informa adecuadamente & $\mathrm{Si}$ & $475(94.8 \%)$ & $433(91,2 \%)$ \\
sobre los medicamentos & $\mathrm{No}$ & $26(5,2 \%)$ & $20(76,9 \%)$ \\
\hline
\end{tabular}

Tabla 1. Características generales de la muestra.

\begin{tabular}{lcc}
\hline \multicolumn{1}{c}{ Clase terapéutica } & Número (\%) & $\begin{array}{c}\text { Numero de medicamentos tomados } \\
\text { simultáneamente (Media } \pm \text { DE) }\end{array}$ \\
\hline Analgésicos/Antiinflamatorios & $367(73,3 \%)$ & $2,59 \pm 1,24$ \\
Anticatarrales & $219(43,7 \%)$ & $2,19 \pm 1,15$ \\
Vitaminas & $120(24 \%)$ & $3,08 \pm 1,26$ \\
Antidepresivos & $67(13,4 \%)$ & $3,30 \pm 1,14$ \\
Antibióticos & $61(12,2 \%)$ & $3,34 \pm 1,24$ \\
Tranquilizantes/Ansiolíticos & $37(7,4 \%)$ & $3,24 \pm 1,36$ \\
Antialérgicos & $34(6,8 \%)$ & $3,41 \pm 1,33$ \\
Anticonceptivos & $24(4,8 \%)$ & $3,38 \pm 1,31$ \\
Otros & $22(4,4 \%)$ & $3,41 \pm 1,33$ \\
\hline
\end{tabular}

Tabla 2. Automedicación por diferentes grupos de medicamentos.

medicamentos, el 10,5\% tomaba cuatro fármacos a la vez, y el $8,8 \%$ tomaba cinco medicamentos. La tabla 2 muestra la media de medicamentos consumidos en función del tipo de medicamento. El número promedio de medicamentos consumidos simultáneamente fue de 2,43 $\pm 1,08$.

Como muestra la tabla 3 , se encontraron diferencias significativas en cuanto a los porcentajes de consumo de cada grupo de medicamentos en función del sexo. Así, el porcentaje de consumidores de antibióticos fue significativamente superior entre los hombres $(22 \%$ frente a $11 \% ; p=0,004$; OR = 1,88; IC95\%: 1,06-3.35; $p=0,031)$. Por otro lado, el porcentaje de consumo de anticatarrales fue superior en mujeres que en hombres (33,9\% en hombres frente a $47,1 \%$ en mujeres; $p=0,012$; OR = 1,70; IC95\%: 1,10-2,63; $p=0,018)$. Los antidepresivos fueron consumidos en mayor número por las mujeres, quedando esta diferencia en el límite de la significación estadística $(8,5 \%$ en varones frente a $14,9 \%$ en mujeres; $p=0,07$; OR $=1,84$; IC95\%: $0,91-3,74 ; p=0,084)$.

La fuente fundamental de obtención de información relacionada con el uso racional del medicamento fue la lectura del prospecto (en un $86,6 \%$ de los casos). Las mujeres presentaron una mayor puntuación relativa a información sobre el medicamento consumido que los hombres $(7,23 \pm 1,93$ en hombres frente a 7,84 $\pm 1,67$ en mujeres, $p=0,002)$. En lo referente a la posible asociación entre el grado de obtención de información sobre el consumo de los diferentes medicamentos (tabla 3), cabe destacar que existió una asociación entre el consumo de antibióticos y el grado de autoinformación sobre los medicamentos relacionada con un uso responsable, presentando los individuos con hábitos de información sobre medicamentos un consumo inferior de antibióticos al de los que poseen escasa información $(11,2 \%$ frente a $21,6 \% ; p=0,03 ;$ OR = 2,15; IC95\%: 1,02-4,51; $p=0,043)$. Cabe comentar finalmente que también las vitaminas fueron consumidas significativamente en mayor medida en los individuos que cursaban estudios sanitarios $(27,8 \%$ frente a $14,6 \% ; p=0,002 ;$ OR = 2,20; IC95\%: 1,32$2,74 ; p=0,003)$.

\section{DISCUSIÓN}

El hábito de automedicación es muy frecuente en nuestra población universitaria. Estas cifras son claramente superiores a las comunicadas en otros estudios en nuestro país. Así, estudios realizados en Alicante y Madrid han arrojado cifras de 63\% y $30,9 \%$ respectivamente ${ }^{2,14}$. Nuestras cifras, pues, se asemejan más a las obtenidas en estudios en población universitaria de Argentina $(85 \%)^{15}$ y de Palestina $(98 \%)^{16}$.

A la cabeza de los medicamentos consumidos se encuentran los analgésicos. Estos datos coinciden 


\begin{tabular}{|c|c|c|c|c|}
\hline Clase terapéutica & Tipo de variable & Número (\%) & P Chi -cuadrado & OR ajustado $(p)$ \\
\hline \multirow{6}{*}{$\begin{array}{l}\text { Analgésicos/ } \\
\text { Antiinflamatorios }\end{array}$} & Sexo masculino & $79(66,9 \%)$ & 0,077 & $1,49(0,085)$ \\
\hline & Sexo femenino & $288(75,2 \%)$ & & \\
\hline & Estudios sanitarios & $258(72,3 \%)$ & 0,433 & $1,16(0,506)$ \\
\hline & Estudios no sanitarios & $109(75,7 \%)$ & & \\
\hline & Buenos hábitos de información & $329(73,4 \%)$ & 0,899 & $0,99(0,965)$ \\
\hline & Poco hábito de información & $38(72,5 \%)$ & & \\
\hline \multirow[t]{6}{*}{ Anticatarrales } & Sexo masculino & $39(33,9 \%)$ & 0,012 & $1,70(0,018)$ \\
\hline & Sexo femenino & $180(47,1 \%)$ & & \\
\hline & Estudios sanitarios & $155(43,8 \%)$ & 0,844 & $1,03(0,888)$ \\
\hline & Estudios no sanitarios & $64(44,8 \%)$ & & \\
\hline & Buenos hábitos de información & $200(44,9 \%)$ & 0,19 & $0,696(0,244)$ \\
\hline & Poco hábito de información & $19(35,3 \%)$ & & \\
\hline \multirow[t]{6}{*}{ Vitaminas } & Sexo masculino & $30(25,4 \%)$ & 0,679 & $0,92(0,737)$ \\
\hline & Sexo femenino & $90(23,6 \%)$ & & \\
\hline & Estudios sanitarios & $99(27,6 \%)$ & 0,002 & $0,450(0,003)$ \\
\hline & Estudios no sanitarios & $21(14,8 \%)$ & & \\
\hline & Buenos hábitos de información & $113(25,1 \%)$ & 0,071 & $0,482(0,086)$ \\
\hline & Poco hábito de información & $7(13,7 \%)$ & & \\
\hline \multirow[t]{6}{*}{ Antidepresivos } & Sexo masculino & $10(8,5 \%)$ & 0,074 & $1,84(0,092)$ \\
\hline & Sexo femenino & $57(14,9 \%)$ & & \\
\hline & Estudios sanitarios & $45(12,6 \%)$ & 0,426 & $1,21(0,493)$ \\
\hline & Estudios no sanitarios & $22(15,3 \%)$ & & \\
\hline & Buenos hábitos de información & $63(14,1 \%)$ & 0,215 & $0,54(0,249)$ \\
\hline & Poco hábito de información & $4(7,8 \%)$ & & \\
\hline \multirow[t]{6}{*}{ Antibióticos } & Sexo masculino & $22(18,6 \%)$ & 0,014 & $0,53(0,031)$ \\
\hline & Sexo femenino & $39(10,2 \%)$ & & \\
\hline & Estudios sanitarios & $49(13,7 \%)$ & 0,095 & $0,57(0,112)$ \\
\hline & Estudios no sanitarios & $12(8,3 \%)$ & & \\
\hline & Buenos hábitos de información & $50(11,2 \%)$ & 0,032 & $2,15(0,043)$ \\
\hline & Poco hábito de información & $11(21,6 \%)$ & & \\
\hline \multirow{6}{*}{$\begin{array}{l}\text { Tranquilizantes/ } \\
\text { Ansiolíticos }\end{array}$} & Sexo masculino & $5(4,2 \%)$ & 0,135 & $2,05(0,148)$ \\
\hline & Sexo masculino & $32(8,4 \%)$ & & \\
\hline & Estudios sanitarios & $26(7,3 \%)$ & 0,89 & $1,04(0,916)$ \\
\hline & Estudios no sanitarios & $11(7,6 \%)$ & & \\
\hline & Buenos hábitos de información & $32(6,9 \%)$ & 0,454 & $1,54(0,398)$ \\
\hline & Poco hábito de información & $5(9,8 \%)$ & & \\
\hline \multirow[t]{6}{*}{ Antialérgicos } & Sexo masculino & $12(10,2 \%)$ & 0,095 & $0,55(0,109)$ \\
\hline & Sexo femenino & $22(5,7 \%)$ & & \\
\hline & Estudios sanitarios & $26(7,3 \%)$ & 0,487 & $0,78(0,545)$ \\
\hline & Estudios no sanitarios & $8(5,3 \%)$ & & \\
\hline & Buenos hábitos de información & $31(6,9 \%)$ & 0,778 & $0,80(0,724)$ \\
\hline & Poco hábito de información & $3(5,9 \%)$ & & \\
\hline \multirow[t]{6}{*}{ Anticonceptivos } & Sexo masculino & $0(0 \%)$ & 0,005 & ** \\
\hline & Sexo femenino & $24(6,3 \%)$ & & \\
\hline & Estudios sanitarios & $16(4,5 \%)$ & 0,611 & $1,13(0,784)$ \\
\hline & Estudios no sanitarios & $8(5,6 \%)$ & & \\
\hline & Buenos hábitos de información & $20(4,5 \%)$ & 0,287 & $2,09(0,204)$ \\
\hline & Poco hábito de información & $4(7,8 \%)$ & & \\
\hline
\end{tabular}

Tabla 3. Diferentes grupos de medicamentos y variables que afectan a su consumo.

ampliamente con los comunicados en otros estudios $^{10,13,15,16}$. Es conocido que el hábito generalizado de consumo de analgésicos y antiinflamatorios puede tener consecuencias serias a nivel hepático en caso de consumo de paracetamol ${ }^{17}$, y sobre la función renal ${ }^{18}$ y el aparato digestivo ${ }^{19}$ en caso de AINEs.
Es de destacar que el hecho de poseer hábitos de información relacionados con el uso responsable del medicamento estaba significativamente asociado a una disminución en el consumo de antibióticos. Es frecuente que individuos que tienen escasos hábitos de información sanitaria en general, y sobre medicamentos en particular, confundan infecciones 
respiratorias víricas con bacterianas, empleando los antibióticos en cuadros víricos que no requieren en principio estos tratamientos. Nuestros datos reafirman la utilidad y propiedad de las campañas estatales de información sobre los riesgos de un consumo indiscriminado e inadecuado de antibióticos.

Por otro lado, cabe destacar la influencia del sexo en el consumo de determinados grupos de medicamentos. De entre todos los productos farmacéuticos diferencialmente consumidos en función del sexo, los antidepresivos pueden ser explicados por la mayor prevalencia en el sexo femenino de la enfermedad que motiva el tratamiento. Mientras que unos autores no han evidenciado estas diferencias de consumo de medicamentos entre sexos ${ }^{15,16}$, otros autores sí han demostrado comportamientos diferenciados según el género en lo tocante a automedicación ${ }^{20}$. Nuestros resultados sobre grado de información acerca de los medicamentos consumidos concuerdan con los obtenidos por estos últimos autores, confirmando el comportamiento automedicativo más responsable del sexo femenino frente al masculino.

Por último cabe comentar que no hemos evidenciado un claro impacto del carácter sanitario de la carrera cursada sobre los hábitos de automedicación. El hecho de que los estudiantes de carreras sanitarias consuman más vitaminas puede justificarse por la mayor carga docente y exigencia de esfuerzo intelectual de las carreras sanitarias contempladas sobre las no sanitarias incluidas en este estudio.

Así pues, la automedicación es una práctica extremadamente frecuente en la población universitaria valenciana estudiada. El sexo y los hábitos de información sobre medicamentos influencian significativamente esta práctica. Creemos que las autoridades sanitarias deben perseverar en su esfuerzo informativo sobre los riesgos de un consumo incontrolado y poco informado de medicamentos.

Como conclusión, la práctica de la automedicación es muy frecuente en población universitaria. El consumo de antibióticos es significativamente inferior en universitarios que cursan carreras estrictamente sanitarias. Las mujeres presentaron un comportamiento más responsable por lo que respecta a la automedicación. Las diferencias en cuanto al tipo de medicamento consumido por sexo en régimen automedicativo son atribuibles a la diferente prevalenvcia de determinadas enfermedades en cada sexo.

\section{BIBLIOGRAFÍA}

1. Gordon SM, Mosure DJ, Lewis J, Brown S, McNagny SE, Schmid GP. Prevalence of self-medication with antibiotics among patients attending a clinic for treatment of sexually transmitted diseases. Clin Infect Dis. 1993; 17:462-5.
2. Bastante T, De la Morena F. Automedicación en población universitaria. XV Congreso de Estudintes de Medicina Preventiva y Salud Pública: Hábitos saludables en el siglo XXI. Facultad de Medicina. Departamento de Medicina Preventiva y Salud Pública. Universidad Autónoma de Madrid. Madrid. España. Junio 2002.

3. Shankar PR, Partha P, Shenoy N. Self-medication and nondoctor prescription practices in Pokhara valley, Western Nepal: a questionnaire-based study. BMC Fam Pract. 2002; 3:17.

4. Blenkinsopp A, Bradley C. Patients, society, and the increase in self medication. Br Med J. 1996; 312:629-32.

5. Laporte JR, Castel JM. El médico ante la automedicación. Med Clin (Barc). 1992; 99:414-6.

6. Honig PK, Guillespie BK. Drug Interactions Between Prescribed and Over the Counter Medication. Drug Safety. 1995;13:296-02.

7. Martins AP, Miranda AC, Mendes Z, Soares MA, Ferreira $P$, Nogueira A. Self-medication in a Portuguese urban population: a prevalence study. Pharmacoepidmiol Drug Saf. 2002;11:409-14.

8. Stoelben S, Krappweis J, Rossler G, Kirch W. Adolescents' drug use and drug knowledge. Eur J Pediatr. 2000; 159:60814.

9. Beitz R, Doren M, Knopf H, Melchert HU. Self medication with over-the-counter (OTC) preparations in Germany. Bundesgesundheitsblatt Genundheitsforschung Gesundheitsschutz. 2004; 47:1043-50.

10. James H, Handu SS, Al Khaja KAJ, Otoom S, Sequeira RP. Evaluation of the knowledge, attitude and practice of selfmedication among first-year medical students. Med Princ Pract. 2006; 15:270-5.

11. Buke C, Hosgor-Limoncu M, Ermertcan S, Ciceklioglu M, Tuncel M, Köse T, et al. Irrational use of antibiotics among university students. J Infect 2005; 51:135-9.

12. Sedighi B, Ghaderi-Sohi S, Emami S. Evaluation of self-medication prevalence, diagnosis and prescription in migraine in Kerman, Iran. Saudi Med J. 2006; 27:377-80.

13. Lau GS, Lee KK, Luk CT. Self-medication among university students in Hong Kong. Asia Pac J Public Health. 1995; 8:153-7.

14. Universidad de Alicante. España. Automedicación y universitarios. La Verdad. 10 de Septiembre de 1998.

15. Aguado M, Nuñez Mb, Dos Santos Antola L, Bregni C. Automedicación en Estudiantes de Farmacia de la Universidad Nacional del Nordeste, Argentina Acta Farm Bonaerense. $2005 ; 24: 271-6$

16. Sawalha AF. A descriptive study of self-medication practices among Palestinian medical and nonmedical university students. Res Social Adm Pharm. 2008:164-72.

17. Bolesta S, Haber SL. Hepatotoxicity associated with chronic acetaminophen administration in patients without risk factors. Ann Pharmacother 2002; 36:331-3.

18. Laporte JR. Automedicación: ¿la información de los usuarios aumenta al mismo tiempo que el consumo? Med Clin (Barc). 1997; 109:795-6.

19. Laporte JR, Carne X, Vidal X, Moreno V, Juan J. Upper gastrointestinal bleeding in relation to previous use of analgesics and non-esteroidal anti-inflammatory drugs. Lancet. 1991; 337:85-9.

20. Abahussain E, Matowe LK, Nicholls PJ: Self reported medication use among adolescents in Kuwait. Med Princ Pract. 2005; 14:161-4. 